cancer led me to believe that here was a splendid archaism, but on reflection I realized that there had been a further enrichment of the English language because of the elision of a hyphen. The editorials are rather pompous and turgid, and censorious of a government which dares to restrict the amount of money put into cancer research. It is not possible to determine how publication time relates to receipt of papers nor is it clear whether or not there is a refereeing system.

The cover of CI displays "Cancer" in big white letters, like its much older antecedent of that name. "Investigation" is added in much smaller black type. As few such journals are likely to attract the casual buyer, such a ploy could hardly be thought deceitful but to a disinterested outsider it seems misleading.

It has always been difficult to define "biological reagents", and the concept of a biological response modifier, apparently coined by Dr Vincent T. DeVita Jr, Director of the National Cancer Institute, is even more complicated. As stated in the Journal of Biological Response Modifiers (JBRM), it includes consideration of

a large number of agents: immunoaugmenting, immunomodulating, and immunorestorative agents; interferon and interferon inducers; lymphokines; cytokines; thymic factors; antitumor and antilymphoid cell antibodies; tumor antigens and modifiers of tumor antigens on cell membranes; antigrowth factors; and maturation and differentiation factors.

Essentially, it means what the Biological Response Modifiers Programme Coordinators want it to mean and anything else for which there is almost a splendid, albeit fictional, precedent. The large editorial board has one or two lonely foreigners known to be sympathetic to the Programme, but the JBRM is so far also predominantly an American effort in

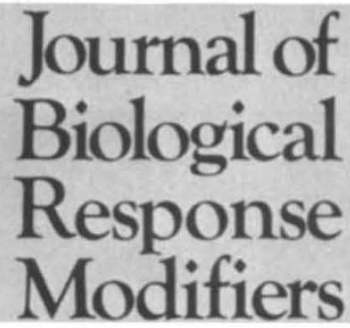

content. The argument is advanced, quite fairly, that pressure on other journals makes the new journal inevitable in the welter of "biologicals" emerging from the monoclonal and genetic engineering technological revolutions. Again, no indication is given of publication times.

There are published here opinions on contentious issues in science. For example, Harold Hewitt is given the opportunity to air his well-known opposition to the tumour immunology package and in a subsequent issue Ronald B. Herberman fires back a broadside against such temerity. The papers make lively reading if one can swallow an initial anxiety at the rag-bag of topics. If the contributions to
JBRM are well refereed the journal could prove to be a useful addition to the literature, and might even act as a quality control device on the burgeoning "biologicals" industry. But it is, I fear, a crusading effort with little chance that impartiality and objectivity will count for much.

K. Hellman and G. Nicolson are prominent members of that part of the scientific community which gives special consideration to the metastasis of cancer cells. Now they have started their own journal, Clinical \& Experimental Metastasis (CEM). There are others on the same topic but this has not proved a

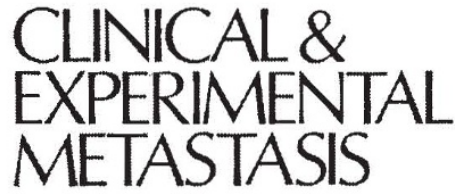

deterrent. A deliberate effort has been taken to make the journal fully international, in that there are representatives on the editorial board from each of a number of countries in which cancer research is funded. The papers published reflect this international flavour. As yet, CEM contains no frills in terms of the airing of controversies, letters to the editor and the like. The illustrations are good, the papers readable and informative. The publication time is difficult to determine on the first few issues but it seems to be coming down from a year or so to about four to six months. It is implied that there is a refereeing system, the submission times being given separately from a time of acceptance. I find it difficult to understand why journals do not state explicitly what their policy is on this important matter. Much of the work of scientists is judged by their output of papers, which in the good old days could reasonably be assumed to be subject to the regulatory influence of studious and independent refereeing. With such a plethora of new journals it is not easy to know if standards are being upheld.

There is little doubt that the technological advances of the past few years will, in time, enable us to control malignant cells in the human body (though the difficulties involved should not be underestimated). All three of the journals will contain a part of the record of progress and they are welcome on this score. But to my mind, well before the story is anywhere near complete, the profligate use of trees to act as the vehicle for scientific pronouncements will have ceased and new information will be surveyed on a television screen. It would have been good to have seen at least a hint that these new journals were preparing for the likely change in medium.

Tony Davies is Chairman of the Section of Biology at the Institute of Cancer Research, London.

\section{Short of practice in immunology}

\section{Gordon Reeves}

Diagnostic Immunology.

Editor Mariano F. La Via.

Alan R. Liss. 4/yr. \$45 (personal); $\$ 90$ (institutional).

THE immunological literature is both extensive and fabulous. The clinical immunologist has the dual task of keeping abreast of advances in immunobiology, immunochemistry, immunogenetics and immunopathology, and of assessing their relevance to the study of human disease. The first requirement is well served by the excellent Annual Review of Immunology and Immunology Today. But what of the practice of clinical immunology? Of the various journals which purport to cover this end of the spectrum, only Clinical and Experimental Immunology, in my view, maintains a consistently high standard. Nevertheless, the need for a journal which seeks to improve the quality and organization of current practice, as identified in the editorial for the first issue of Diagnostic Immunology (DI), is probably a real one.

Unfortunately, little of the content of the first four issues of DI concerns current clinical practice. Although the pitch is often research-and-development orientated, much of this material compares unfavourably with papers appearing in the well-established Journal of Immunological Methods. Two useful reviews have appeared on clinical applications of FACS techniques and immunodiagnosis in protozoan and helminthic infections, but there has been little discussion of the practical value of existing methods and ways of delivering a clinical immunology service. Where are the critiques which review how to diagnose and monitor those disorders falling within the prime areas of clinical immunology, i.e. allergy, autoimmunity, immunodeficiency, lympho-proliferative disease and transplantation? The aim to provide "critical evaluation of reagents, quality assurance, standardization of methods and definition of normal ranges"' has not been fulfilled. The emphasis of the third issue is on the "goal of providing a forum for discussion of new and promising methodologies", whereas the fifth issue (Vol. 2, No. 1) is the first one to contain a reasonable sprinkling of clinically relevant papers.

To me it seems that DI has undergone an identity crisis. Consistency of content, style and production are important prerequisites, if it is to succeed.

Gordon Reeves is Consultant Immunologist and Senior Lecturer at the University Hospital, Queen's Medical Centre, Nottingham. 